\title{
THz Sommerfeld wave propagation on a single metal wire
}

\author{
Tae-In Jeon, ${ }^{\text {a) }}$ Jiangquan Zhang, and D. Grischkowsky ${ }^{\text {b) }}$ \\ School of Electrical and Computer Engineering, Oklahoma State University, Stillwater, Oklahoma 74078
}

(Received 20 December 2004; accepted 2 March 2005; published online 12 April 2005)

\begin{abstract}
We report an experimental and theoretical study of $\mathrm{THz}$ Sommerfeld wave propagation on a single copper wire. THz pulses are optoelectronically generated and launched onto 0.52-mm-diam copper wire, and the guided $\mathrm{THz}$ pulses are detected at the end of the wire by a standard photoconductive antenna. Very low attenuation and group velocity dispersion are observed, and the measured radial field amplitude of the Sommerfeld wave is inversely proportional to the radial distance. These results are consistent with theoretical predictions. Experimental results from curved wires show the weakly guiding property of the THz Sommerfeld wave, which will limit its applications. (C) 2005 American Institute of Physics. [DOI: 10.1063/1.1904718]
\end{abstract}

There has been increasing interest on the guided wave propagation of $\mathrm{THz}$ pulses, and much effort and progress on $\mathrm{THz}$ waveguide study have been made in the past few years. ${ }^{1-4}$ Among various types of $\mathrm{THz}$ waveguides, parallelplate metal waveguides have attracted considerable attention due to the low loss and low group velocity dispersion in the transverse-electromagnetic (TEM) mode propagation. ${ }^{2-4}$ For a parallel-plate copper waveguide with $100 \mu \mathrm{m}$ plate separation, the typical frequency-dependent amplitude absorption coefficient caused by the finite conductivity of copper is approximately $0.06 \mathrm{~cm}^{-1}$ at $0.5 \mathrm{THz},{ }^{2-4}$ and is proportional to the square root of frequency and inversely proportional to the plate separation. Quasioptic coupling has been the primary coupling mechanism in these studies, where the $\mathrm{THz}$ pulses are coupled into and out of the waveguides by hyperspherical or plano-cylindrical silicon lenses. In a recent report, sub-mm coaxial lines have been characterized for the TEM mode propagation of $\mathrm{THz}$ pulses. ${ }^{5}$ In that report, instead of using quasioptic coupling, optoelectronically generated $\mathrm{THz}$ pulses are directly launched into the coaxial lines, and the propagated pulses are then optoelectronically detected by a standard photoconductive $\mathrm{THz}$ receiver. The coaxial line solves the diffraction problem in the unguided dimension of the parallel-plate waveguides; however, the absorption is higher than that for parallel-plate waveguides. 5

Single metal wires have been theoretically and experimentally studied for guided propagation of electromagnetic waves from tens of meters to millimeter waves. ${ }^{6-10}$ On a cylindrical conductor (wire), electromagnetic waves propagate as weakly guided surface waves, due to the finite conductivity of the metal. This type of guided propagation does not occur with a perfect conductor. ${ }^{8}$ The principle mode (the Sommerfeld wave) of such a structure has remarkably low loss and low group velocity dispersion, while all other modes vanish almost immediately upon excitation due to their high attenuation. ${ }^{6}$ Recently, observations of $\mathrm{THz}$ pulses propagating on single metal wires have been reported, ${ }^{11,12}$ which showed very low loss and low group velocity dispersion.

In this letter, we identify the recently observed $\mathrm{THz}$ pulse propagation on a single wire, ${ }^{11,12}$ as the Sommerfeld

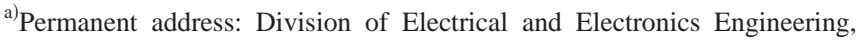
Korea Maritime University, Busan, Korea.

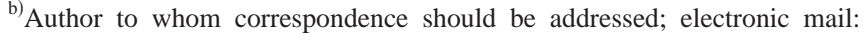
grischd@ ceat.okstate.edu
}

wave, ${ }^{6-10}$ calculate the very low absorption of this wave, and measure the propagation properties. Commercial copper wires of $0.52 \mathrm{~mm}$ diameter and various lengths are used for this study. Unlike the direct generation and launching of $\mathrm{THz}$ pulses in the previous report, ${ }^{5}$ here the $\mathrm{THz}$ pulses are optoelectronically generated by a specially designed $\mathrm{THz}$ transmitter chip, and then directly coupled onto the metal wire. The propagated $\mathrm{THz}$ pulses are detected by a standard photoconductive $\mathrm{THz}$ receiver. ${ }^{5}$ Figure 1(a) shows the $\mathrm{THz}$ transmitter used in this experiment, which is composed of two concentric electrodes deposited on a silicon-on-sapphire (SOS) chip. The inner electrode is an $80-\mu \mathrm{m}$-diam disk, while the outer electrode has an inner diameter of $260 \mu \mathrm{m}$ with a $30^{\circ}$ opening for the connecting wire of the inner electrode. In the experiment, the inner electrode is biased at $+60 \mathrm{~V}$ with respect to the outer one. The $800 \mathrm{~nm}, 60 \mathrm{fs}$ laser excitation pulses with an average power of $30 \mathrm{~mW}$ and a repetition rate of $83 \mathrm{MHz}$ are focused onto the outer side of the inner electrode to generate $\mathrm{THz}$ pulses. The tip of the copper wire has been carefully polished into a 1-cm-long cone shape with an $80-\mu \mathrm{m}$-diam flat end. The $80 \mu \mathrm{m}$ tip is placed $5 \mu \mathrm{m}$ away from the transmitter chip for optimal $\mathrm{THz}$ coupling. For the detection of the propagated $\mathrm{THz}$ pulses, the polished flat output face of the copper wire, covered by a $14-\mu \mathrm{m}$-thick polyethylene insulation tape, is placed in contact with the THz receiver chip. ${ }^{5}$ As shown in Fig. 1(b), the SOS receiver antenna is between $10 \mu \mathrm{m}$ lines separated by $200 \mu \mathrm{m}$; the photoconductive gap of the antenna is $5 \mu \mathrm{m}$. The antenna is excited by the laser detection pulses with an
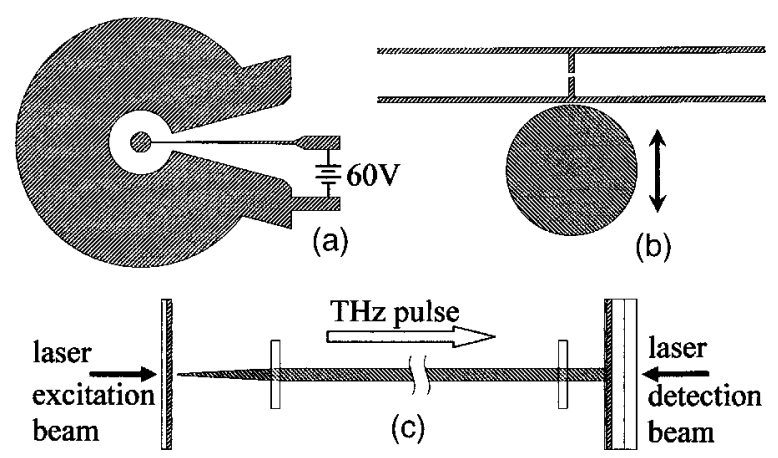

FIG. 1. (a) Schematic diagram of the transmitter chip. (b) The receiver antenna and the output face of the copper wire. (c) Cross section of the transmitter chip, the copper wire, and the detection chip. 

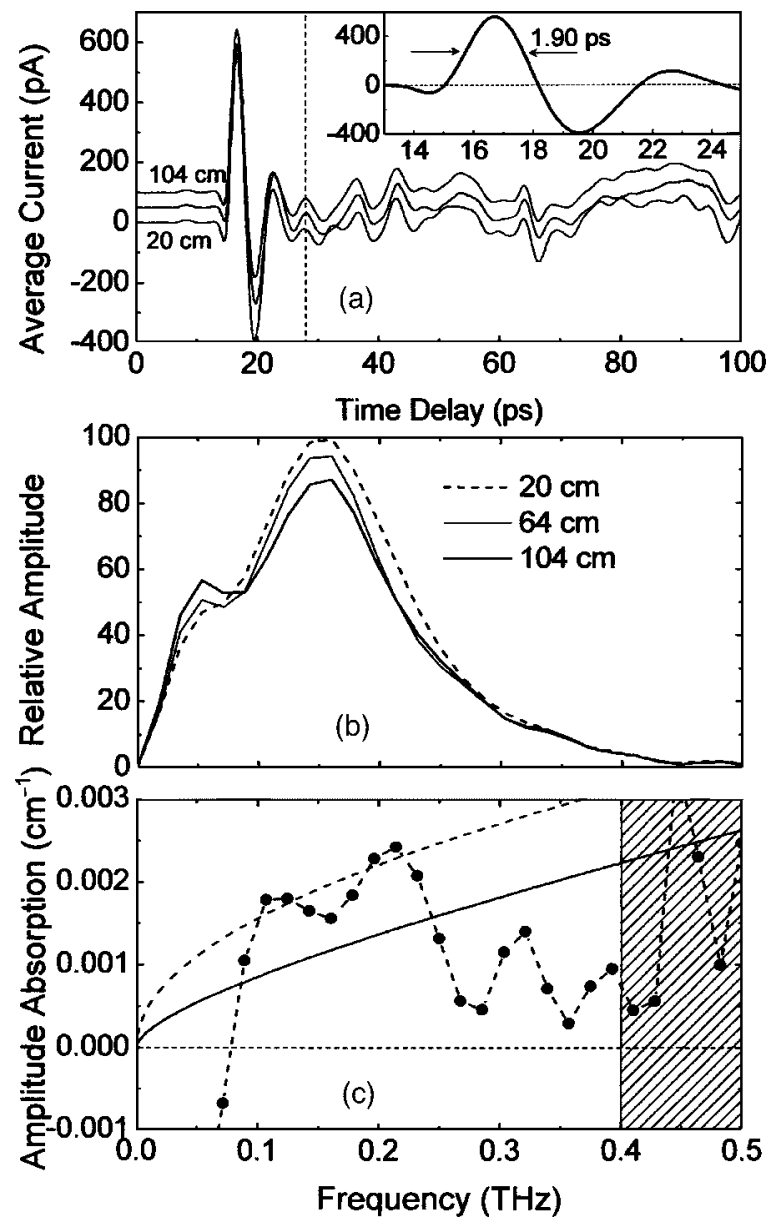

FIG. 2. The transmitted THz pulses for $20 \mathrm{~cm}, 64 \mathrm{~cm}$, and $104 \mathrm{~cm}$ copper wires. The pulses have been shifted for clarity. The inset shows the enlargement of the main peak of the $20 \mathrm{~cm}$ pulse. (b) The spectra of the pulses truncated at the dashed line shown in (a). (c) Amplitude absorption coefficients. Dots: measured from $104 \mathrm{~cm}$ and $20 \mathrm{~cm}$ copper wire (the measurement in the shaded area is considered not accurate); solid line: Sommerfeld theory for a 0.52 -mm-diam copper wire; dashed line: theory for TEM mode of the corresponding air-filled coaxial waveguide.

average power of $10 \mathrm{~mW}$, enabling the measurement of the propagated $\mathrm{THz}$ pulses, by changing the relative time delay between the excitation and detection pulses. ${ }^{1-5}$ As shown in Fig. 1(c), the wire is supported by two tightly fitting Teflon disks of $3 \mathrm{~mm}$ thickness. These disks enable the fine adjustment of the position of the wire for optimal coupling. Sapphire plates of various thicknesses are placed on the back of both the transmitter and receiver chips to remove undesirable reflections of the $\mathrm{THz}$ pulses.

The measured propagated $\mathrm{THz}$ pulses guided by the 20cm-, 64-cm-, and 104-cm-long copper wires are displayed in Fig. 2(a); the pulses have been shifted for clarity. They consist of distinct main peaks and complicated following structures, probably caused by electrical ringing in the transmitter and $\mathrm{THz}$ reflections. The main peaks are almost identical, demonstrating the very low attenuation and low group velocity dispersion of the Sommerfeld wave. The inset is the enlargement of the main peak of the $20 \mathrm{~cm}$ pulse, showing the $1.90 \mathrm{ps}$ full width at half maximum.

Figure 2(b) shows the corresponding spectra obtained by truncating the THz pulses at the dashed line in Fig. 2(a). The amplitude absorption coefficient calculated from the 20 and $104 \mathrm{~cm}$ spectra is plotted in Fig. 2(c), showing the very low propagation loss compared with other types of $\mathrm{THz}$
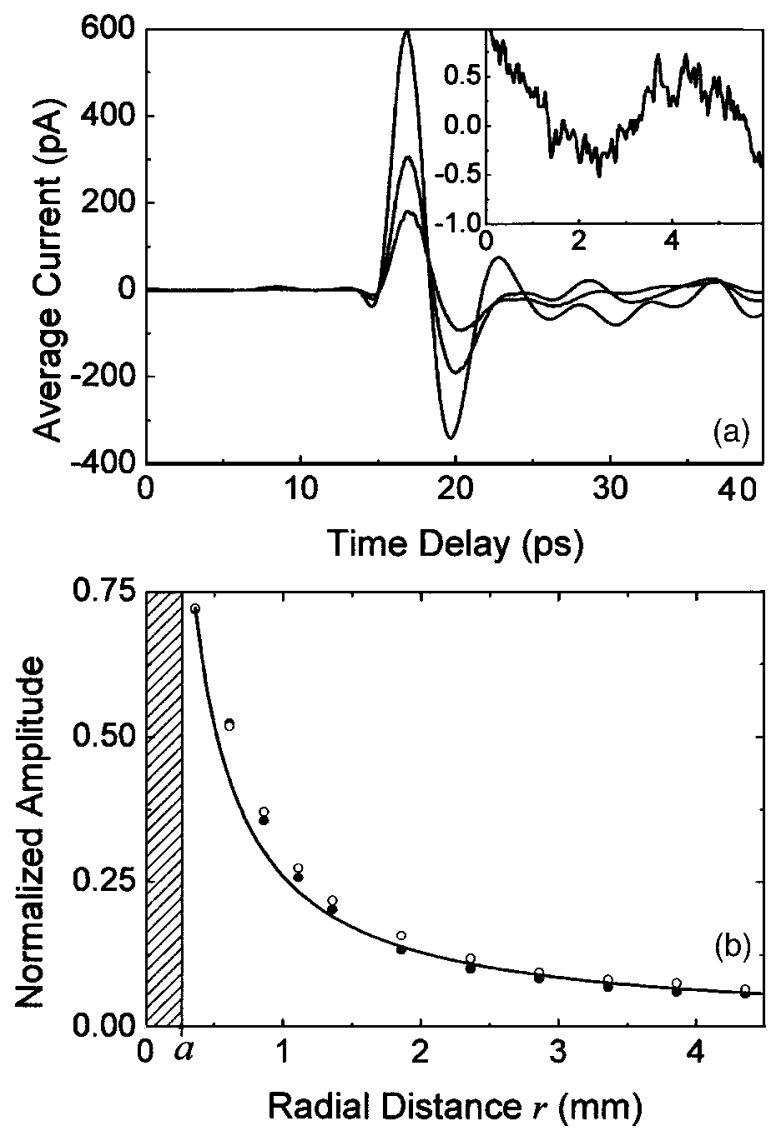

FIG. 3. (a) Transmitted THz pulses for radial distances of 0.36, 0.86, and $1.36 \mathrm{~mm}$ (main peaks from top to bottom). The inset is the leading edge of the $0.36 \mathrm{~mm}$ pulse from 0 to $6 \mathrm{ps}$. (b) Normalized peak amplitude of the pulses (open circles) and normalized spectral amplitude (solid dots) at 0.15 THz. The solid line is the $a / r$ relation. The shaded area represents the cross section of the wire with radius $a=0.26 \mathrm{~mm}$.

waveguides. ${ }^{1-5}$ For comparison, we plot as the solid line the calculated amplitude absorption coefficient for the Sommerfeld wave, and as the dashed line the calculated absorption for the TEM mode of the corresponding air-filled copper coaxial waveguide. This coaxial waveguide would have an inner diameter of $0.52 \mathrm{~mm}$ and an outer diameter of $9 \mathrm{~mm}$, giving the same $a / r$ field dependence and boundaries as shown in Fig. 3(b). Such a corresponding waveguide would be difficult to fabricate and to utilize, and would not have single mode propagation in our frequency range. The cutoff frequency of the $\mathrm{TE}_{11}$ mode would be only $20 \mathrm{GHz}$.

Unlike in the cases of other types of $\mathrm{THz}$ waveguides, an analytical expression for the attenuation of the Sommerfeld wave propagating on a metal wire with finite conductivity is not obtainable, and one has to numerically solve the eigenvalue equation for the complex propagation constant. ${ }^{6-10}$ For a metal wire with conductivity $\sigma_{c}$ and radius $a$, the eigenvalue equation can be written as $H_{0}^{(1)}(\gamma a)$ $/ H_{1}{ }^{(1)}(\gamma a)=i k^{2} / \gamma k_{c},{ }^{10}$ where $H^{(1)}$ is the Hankel function of the first kind, $k$ is the free-space wave number, $k_{c}$ $=\left(\omega \mu_{c} \sigma_{c}\right)^{1 / 2} \exp (-i \pi / 4), \mu_{c}$ is the permeability of the metal, $\gamma^{2}=k^{2}-h^{2}$, and $h$ is the complex propagation constant. The amplitude absorption coefficient is determined by the imaginary part of $h$. With $a=0.26 \mathrm{~mm}$, and $\sigma_{c}=5.8$ $\times 10^{7} \Omega^{-1} \mathrm{~m}^{-1}$ for copper, the calculated amplitude absorption coefficient is the solid curve in Fig. 2(c).

From Fig. 2(c), the measured attenuation of the $\mathrm{THz}$ Sommerfeld wave is comparable with the calculated one. 


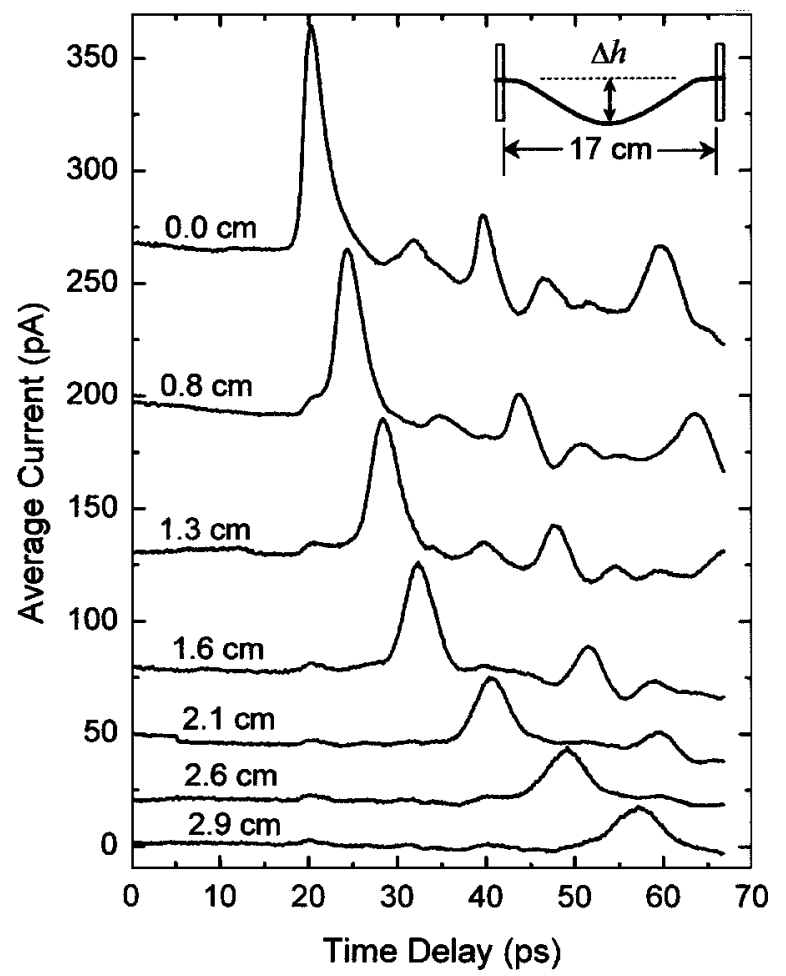

FIG. 4. Transmitted THz pulses for the indicated curve depths $\Delta h$. The inset shows the curved wire.

The systematic experimental errors appear to be of the same order as the very low attenuation predicted by theory. Therefore, the experimental results are not considered to be an accurate measurement of the absorption, but clearly demonstrate the remarkably low attenuation of the $\mathrm{THz}$ Sommerfeld wave.

To understand the radial field pattern of the Sommerfeld wave, which has no angular dependence, we measured the transmitted pulses at different radial distances from the surface of a 20-cm-long copper wire. The transmitter and receiver chips were fixed, while the output end of the wire was moved vertically relative to the receiver antenna to change the radial separation between the wire and the antenna, as indicated by the arrows in Fig. 1(b). The THz pulses obtained for radial distances of $0.36,0.86$, and $1.36 \mathrm{~mm}$ are displayed in Fig. 3(a), with the excellent signal-to-noise ratio of $\sim 3000$, as documented by the high frequency noise level in the inset. The larger slow oscillations shown in the inset are reproducible and are considered to be optical reflections from the various surfaces of the optical beam train. Figure 3(b) shows both the normalized peak amplitude of the pulses (open circles) and the normalized spectral amplitude at 0.15 $\mathrm{THz}$ (solid dots) as functions of the radial distance. The radial component of the electrical field outside the wire can be expressed as $E_{r}(r) \propto H_{1}{ }^{(1)}(\gamma r) / H_{1}{ }^{(1)}(\gamma a),{ }^{6,10}$ where $r>a$ is the radial distance. When $r<100 a$, the above ratio of the Hankel functions can be approximated as $a / r,{ }^{8}$ i.e., $E_{r}(r)$ $\propto a / r$ when $r<100 a$. It can be seen in Fig. 3(b) that, the experiment acceptably matches the $a / r$ dependence.

For completeness of this study, we also investigated the effect of wire bending on the guiding property of the metal wire. A 18.9-cm-long copper wire was used in this experiment, and the separation between the two Teflon disks was $17 \mathrm{~cm}$. In the experiment the receiver and the output face of the wire were kept fixed, but the transmitter and input face of the wire were moved toward the receiver so that a curve was formed in the middle of the wire, as indicated by the inset in Fig. 4. The transmitted pulses for various curve depths $\Delta h$ show a rapid signal drop with increased wire curvature; the main peak for the $2.9 \mathrm{~cm}$ curve drops to below $1 / 5$ of the original amplitude for the straight wire. These results show the weakly guiding property of the Sommerfeld wave, in agreement with previous work. ${ }^{8,9}$ This weakly guiding property will limit practicable applications.

In summary, we have experimentally and theoretically studied the $\mathrm{THz}$ Sommerfeld wave on commercial copper wires. Our experiment shows the low attenuation and low group velocity dispersion for the $\mathrm{THz}$ Sommerfeld wave, consistent with the early low frequency and microwave experimental and theoretical results, ${ }^{6-10}$ and the recent observations of single wire $\mathrm{THz}$ pulse propagation. ${ }^{11,12}$ The measured radial $\mathrm{THz}$ field amplitude shows the predicted $1 / r$ falloff. We also demonstrated the weakly guiding nature of the $\mathrm{THz}$ Sommerfeld wave.

The authors thank Sharmila Rajendran for the creative transmitter mask design and Utish Rajkarnikar for his experimental assistance. This work was partially supported by the National Science Foundation, the U.S. Army Research Office, and the Semiconductor Research Corporation, Center for Advanced Systems Technologies.

${ }^{1}$ G. Gallot, S. P. Jamison, R. W. McGowan, and D. Grischkowsky, J. Opt. Soc. Am. B 17, 851 (2000).

${ }^{2}$ R. Mendis and D. Grischkowsky, Opt. Lett. 26, 846 (2001).

${ }^{3}$ R. Mendis and D. Grischkowsky, IEEE Microw. Wirel. Compon. Lett. 11, 444 (2001).

${ }^{4}$ S. Coleman and D. Grischkowsky, Appl. Phys. Lett. 83, 3656 (2003).

${ }^{5}$ T.-I. Jeon and D. Grischkowsky, Appl. Phys. Lett. 85, 6092 (2004).

${ }^{6}$ A. Sommerfeld, Electrodynamics (Academic, New York, 1952), pp. 177190.

${ }^{7}$ J. A. Stratton, Electromagnetic Theory, (McGraw-Hill, New York, 1941), pp. 524-537.

${ }^{8}$ G. Goubau, J. Appl. Phys. 21, 1119 (1950).

${ }^{9}$ F. Sobel, F. L. Wentworth, and J. C. Wiltse, IRE Trans. Microwave Theory Tech. 9, 512 (1961).

${ }^{10}$ M. J. King and J. C. Wiltse, IRE Trans. Antennas Propag. 10, 246 (1962).

${ }^{11}$ K. Wang, A. Barkan, and D. M. Mittleman, Appl. Phys. Lett. 84, 305 (2004).

${ }^{12}$ K. Wang and D. M. Mittleman, Nature (London) 432, 376 (2004). 